\title{
Does The Standard Toxicological Testing Paradigm for Industrial Chemicals Apply to Screening for Children's Health Risks?
}

\author{
Laura Plunkett ${ }^{1}$ and Richard A. Becker ${ }^{2, *}$ \\ ${ }^{1}$ Integrative Biostrategies, LLC, 1223 Melford Drive, Houston, TX 77077, USA and ${ }^{2}$ American Chemistry Council, 1300 \\ Wilson Blvd., Arlington VA 22209, USA
}

\begin{abstract}
Questions have been raised concerning whether standard toxicological testing paradigms for characterizing hazards are applicable for children. In this paper, the standard toxicological testing paradigm for industrial chemicals ${ }^{1}$ is examined. The analysis includes examination of the basic principles and elements of hazard characterization methodology based on animal toxicity data, examination of the characteristics of the standard toxicity tests that apply to assessing hazards of industrial chemicals to children's health, and an appraisal of specific industrial chemical hazard characterizations developed by USEPA covering more than 200 substances (High Production Volume (HPV) Challenge datasets ${ }^{2}$ ) with regard to their relevancy for use in screening for potential risks to children's health. We conclude that the standard toxicity tests used for industrial chemicals that comprise the USEPA's HPV Challenge provide adequate information for developing screening-level hazard characterizations for children's health, and when coupled with child-specific exposure information should provide adequate screening-level risk evaluations for children.
\end{abstract}

Key Word: Children's health, HPV chemical, hazard characterization, toxicity testing, industrial chemicals.

\section{INTRODUCTION}

In the last few years, as evidenced by the number of papers appearing in scientific journals, there has been a large increase in research dealing with the differences between children and adults in terms of both their potential for exposure to environmental chemicals and their response to chemical exposure (i.e., toxicity). Concomitantly, children's health has become an important focus of regulatory initiatives within the United States, due in part to an increased awareness by the public of the inherent differences in physiology and behavior patterns between children and adults. Child-focused regulatory hazard and risk assessment initiatives have been launched by both the U.S. Food and Drug Administration (USFDA) and the U.S. Environmental Protection Agency (USEPA), and are examples of the recent increase in regulatory focus on children's health. In 1998, the USFDA passed regulations to encourage increased testing of prescription drugs in pediatric populations as a way not only to improve physicians' understanding of how to prescribe drugs for children in terms of therapeutic efficacy,

\footnotetext{
*Address correspondence to this author at the American Chemistry Council, 1300 Wilson Blvd., Arlington VA 22209, USA; Tel: 703/741-5000; Fax: 703/741-6210; E-mail: rick_becker@americanchemistry.com
}

${ }^{1}$ In this paper, "industrial chemicals" will refer to chemicals used in manufacturing of commercial and consumer products, and not chemicals developed solely as pharmaceuticals, food additives or pesticides.

${ }^{2}$ Under the HPV Challenge program, companies provide health and environmental effects information to EPA (for evaluation by the Agency and for public dissemination) for chemicals produced or imported in the United States in quantities of 1 million pounds or more per year. Companies have sponsored more than 2,200 HPV chemicals, of which approximately two-thirds of the chemicals are proceeding through the U.S. HPV Challenge Program and approximately $1 / 3$ are proceeding through international efforts such as the OECD Refocused HPV Chemicals Programme. but also as a way to ensure that drugs are used safely in children [1]. During the same time period, to address increasing concerns over potential risks to children's health from exposures to trace levels of environmental chemicals, the USEPA implemented the Food Quality Protection Act (US Public Law 104-170) [2], focusing on potential risks to children from exposures to pesticides. In a recent document, the World Health Organization (WHO) has set forth a series of recommendations as principles for evaluating children's health risks associated with exposure to environmental chemicals [3], indicating the focus of regulatory agencies worldwide on collection of data to address the potential health risks to children from exposure to chemicals in their environment, particularly in developing countries.

The USEPA also launched a pilot program, the Voluntary Children's Chemical Evaluation Program (VCCEP), concentrating on chemicals that children could be exposed to from industrial, commercial and consumer product sources [4]. This child-focused initiative is a voluntary program instituted by USEPA's Office of Prevention, Pesticides and Toxic Substances (OPPTS), and was only developed after extensive stakeholder dialog and public meetings over the course of more than a year. Participants in the public meetings included industry, children's health and environmental groups, animal welfare groups, and others. The VCCEP, which follows a risk assessment paradigm (integrating hazard data with exposure information), is a tiered evaluation program aimed at evaluating and presenting the potential risks of certain chemicals to children. The VCCEP's pilot program (currently ongoing) also includes a unique evaluation step at each tier, where the hazard data and exposure information submitted by the sponsor are critically reviewed by a group 
of scientific experts with extensive and broad experience in toxicity testing and exposure evaluations.

A tiered testing framework that is based on the VCCEP model was recently described by Becker et al. [5]. In the tiered framework, each safety assessment is designed to begin with the same endpoints (using the harmonized set of toxicity tests) evaluated under the Organization for Economic Cooperation and Development (OECD) Screening Information Data Set SIDS $^{3}$ and High Production Volume (HPV) Challenge programs (described below), and then, when necessary, to build from the basic data sets in a tiered fashion. This represents a knowledge-based approach, whereby information gained from specific toxicity studies is used to evaluate whether there is a need for additional laboratory toxicity tests and to indicate which specific type or types of toxicity tests should be conducted.

Underlying each of the regulatory-based children's health initiatives is the perception that relatively little is known about the toxic properties of chemicals, in particular industrial chemicals. With respect to public availability of HPV toxicity information, prior to initiation of the HPV Challenge Program, it was reported that more than half of the HPV chemicals had never been tested for potential toxicity [6]. Just as the HPV Challenge started, EPA reported that, in terms of publicly available data, $43 \%$ of the HPV chemicals had no testing data on basic toxicity, and only $7 \%$ had a full set of basic test data. Furthermore, for approximately $75 \%$ of the HPV chemicals, EPA reported there was no screening information available to the public in Agency files for developmental toxicity [7]. However, the limitations of such statements were not fully appreciated. In most cases, these perceptions and statistics were derived from limited reviews that focused on a selected set of databases (i.e., those which could be searched electronically at the time), and sought information on only certain specific tests. The results of the first review were released by Environmental Defense in mid1997. That study, which acknowledged that a review of a limited number of data sources and endpoints for a sample of 100 HPV chemicals (substances manufactured or imported into the U.S. in quantities exceeding a million pounds per year) concluded that sufficient screening-level toxicity data were not publicly available for many of the HPV chemicals [8]. Subsequently, in 1998, the American Chemistry Council (ACC) [9] and USEPA [10] each produced separate studies of all 2,800 HPV chemicals, and independently found publicly available data for only about 20 percent of the screening tests recommended by the OECD. These reviews, however, did not, and could not examine all likely sources of data on chemical toxicity. The reason is that the vast majority of hazard data on such chemicals was not maintained in publicly available, electronically searchable databases or published in scientific journals. Historically, such data has been generated and maintained by manufacturers in support of their product safety assessments, occupational hygiene programs, and other activities. When required by regulation,

${ }^{3}$ The OECD created the Screening Information Data Sets program, commonly known as "SIDS," to secure uniform sets of hazard-screening information on industrial chemicals worldwide. The OECD SIDS standards comprise a series of data sets, tests, testing protocols, and information formats for conducting basic hazard assessments of industrial chemicals. the data are submitted to various government agencies and databases. One of the goals of the HPV Challenge Program and the pilot VCCEP program has been to make more of the existing data, and other important information about chemicals, publicly available.

When the USEPA challenged the chemical industry in 1998 to generate OECD SIDS-level hazard screening data sets for HPV chemicals, under the HPV Challenge Program, companies formally committed to gather and make publicly available existing SIDS-level screening data on HPV chemicals. Where adequate data or other information did or does not exist, new tests have been or will be conducted. More than 2,200 chemicals have been included in this effort, which included an agreement to make the results publicly available. For each of the HPV chemicals sponsored in the program, sponsors provide 17 types of information, including summarized results in four categories: physical-chemical properties, environmental fate, and potential to induce toxicity in aquatic organisms and humans. Data to be summarized for human toxicity include studies assessing acute toxicity, subchronic toxicity, genotoxicity, and developmental and reproductive toxicity. The information required for human health hazard assessment in the HPV Challenge Program is identical to the internationally-agreed SIDS standards, established by the 30 nations of the OECD. Given the recent heightened focus on children, we have asked, "Does the standard toxicological testing paradigm for industrial chemicals apply to screening for children's health risks?" and in this paper we map out areas where these standard tests apply to the hazard characterization elements of child-focused risk evaluations.

\section{METHODS OF ANALYSIS}

The analysis began first by examining the principles and overarching elements of hazard characterization and doseresponse evaluation that impact confidence in the outcome of a screening-level risk assessment for children's health. Next, the types of toxicity tests used to gather data for HPV chemicals are reviewed and those tests (or characteristics of the tests) that apply to assessing hazards to children's health are described. Then, the HPV Challenge information is reviewed and the specific hazard characterizations developed by USEPA from HPV Challenge datasets are discussed with regards to their relevancy for use in screening for potential risks to children's health.

\section{Routine Toxicity Tests: What Do They Teach Us?}

Contrary to suggestions that have appeared in the popular and scientific press, current toxicity testing paradigms for drugs, food, pesticides, industrial chemicals and even medical devices provide important and useful information relevant to assessing risks to infants and children, as well as the developing fetus. First and foremost is the basic principle of toxicology and risk assessment that results of toxicity tests in animals are predictive of effects expected in humans of all ages [11]. Although there may be species differences, in the absence of empirical data, hazard characterizations assume relevancy of the animal tests to humans, and there is a solid basis of biomedical scientific literature to support the healthprotective nature of this assumption. Furthermore, current USEPA risk assessments for chemicals assume that vulner- 
able subgroups are protected when risk assessments are performed based on standard toxicity tests [12].

It must be remembered as well that susceptibility to chemicals is not only an issue of age (e.g., child versus adult). Factors such as genetics, gender, ethnicity, nutritional status, and behavior or lifestyle all contribute to the differences seen in responses to chemicals among individuals [13]. Thus, the standard of practice is to employ toxicity tests in laboratory animals to identify target sites of toxicity and dose-response information for hazard assessment applicable to a range of subpopulations of concern, including children. Within the battery of standardized toxicity tests employed for hazard characterization, specific study designs and endpoints are included to cover concerns regarding dose, target organs, and developmental windows of susceptibility. Becker et al. [5] showed that integrating toxicity decision triggers within a tiered toxicity testing and evaluation framework can provide an efficient and effective approach to develop data for hazard characterizations.

An area of risk assessment (and hazard characterization) that is often overlooked in discussions of susceptible populations is how the standard practice of employing uncertainty factors in risk assessments contributes to health protection. Once toxicity testing has identified effect levels or hazard levels for particular chemicals in animals, it is standard practice to apply or employ uncertainty factors (sometimes referred to as safety factors), modifying factors, or modeling to extrapolate from responses in laboratory animals to responses expected in humans. These factors are designed to span the breadth of vulnerability in the human population. The most common application of such safety factors is a factor of 10 to account for intra-species variability (differences between individuals of the same species) followed by another factor of 10 to account for inter-species extrapolation (animal to human). The logic behind the use of such safety factors is to assure that all vulnerable subgroups, including children, are protected. The International Programme on Chemical Safety (IPCS) [14] indicates the interspecies extrapolation factor can be subdivided into components (a 4fold factor for toxicokinetics and 2.5-fold toxicodynamics), and the inter-individual extrapolation factor can be divided evenly (3.3-fold for both kinetics and dynamics). If uncertainties have been adequately addressed in all areas, a composite safety factor of 1 is used. Extra uncertainty factors, which are not part of these general extrapolation safety factors, are used to address limitations in the toxicity dataset of a given substance. If uncertainties are large, additional factors of 10 are used in a multiplicative manner to form a composite uncertainty factor. Generally, the composite uncertainty factor will not exceed 10,000; it is felt that if the information is so uncertain, then a quantitative approach should typically not be used [15]. Application of safety or uncertainty factors, therefore, is one way that toxicologists and risk assessors have approached the issue of assuring protection of vulnerable subgroups such as children when interpreting animal toxicity data and applying their results to human populations.

Another area in the discussion of toxicity testing and health protection, for adults as well as children, is dose selection in animal testing. For hazard characterization, inherent limitations in animal models and toxicity testing protocols in terms of sensitivity are addressed by using high doses. Animal model systems employed in standard toxicity testing routinely employ dose levels that are, 100-, 1,000- or even 10,000 -fold higher than humans would be expected to experience.

In fact, in order to provide assurance that potential toxicity will not be missed, the standard toxicity testing protocols for reproductive and developmental toxicity testing all require that the highest dose tested be chosen with the aim to induce some developmental and/ or maternal toxicity but not death or severe suffering $[16,22]$. While this approach is precautionary toxicology, because it ensures that there is little chance of "missing" a potential adverse effect, it also has the consequence of complicating communication efforts and precludes use of simple descriptors. Adverse reproductive or developmental effects observed at dose levels that produce parental toxicity may be secondary effects. If studies are conducted under conditions of overt toxicity, such effects may not be indicative of unique or selective developmental or reproductive toxicity. As a result, the only way to adequately communicate potential hazards of exposures is in a risk context. This means that the evaluative process must compare the dose-response data generated in the toxicity studies to estimated levels of human exposure to derive a margin of exposure (MOE). The MOE expresses the magnitude of the difference between a level of anticipated human exposure and the highest level at which there is no significant increase in the frequency of an adverse effect. This is critical information not only for assessing risk and considering risk management options when warranted, but also for communicating potential risks to the public.

A careful review of standard toxicity testing protocols typically employed for industrial chemicals, including those used for evaluating HPV chemicals, reveals that data collected with these tests are wide-ranging and relevant not only to assessing risks posed to adults but to humans of various ages and backgrounds. This is because the endpoints examined in the standard toxicity tests are numerous, involve all of the major organ systems, examine both acute and repeated exposures, and involve animals of different ages and stages of development (pre-natal to adult). The standard toxicity testing battery suggested by OECD and USEPA for HPV chemicals involves acute toxicity testing in rodents, repeat dose or subchronic toxicity testing in rodents, genotoxicity testing and reproductive and developmental toxicity testing [17]. While this battery defines the core screening test for HPV chemicals, in many cases the toxicity information data base for important industrial chemicals is much more expansive and includes studies in multiple species and more complex toxicity studies, such as chronic toxicity/carcinogenicity testing in rodents. Standard protocols for acute, subchronic, genetic, and reproductive/developmental toxicity tests are discussed and explained in many toxicology texts including the CRC Handbook of Toxicology [18], Hayes' Principles and Methods of Toxicology [19], as well as guidance documents available from regulatory agencies such as the USEPA (e.g., USEPA OPPTS Harmonized Test Guidelines) [20], the USFDA [21], and the OECD [22]. Controlled toxicology studies in laboratory animals provide the scientific basis for establishing causal relationships between exposure to an agent and various forms of toxicity. Toxicity tests for chemicals performed for regulatory submittal are conducted in 
Table 1. Symptoms and Signs Routinely Evaluated in Toxicity Tests in Laboratory Animals

\begin{tabular}{|c|c|}
\hline respiratory effort or rate changes & ocular system effects (e.g., color, excretions) \\
\hline motor activity & cardiovascular signs and symptoms \\
\hline convulsions & salivation \\
\hline reflexes & piloerection \\
\hline signs of analgesia & gastrointestinal system changes \\
\hline muscle tone & skin appearance \\
\hline paralysis & limb splay \\
\hline lethargy & hypersensitivity \\
\hline tail elevation & tremor \\
\hline spasticity & behavioral changes \\
\hline
\end{tabular}

accordance with standardized protocols following regulatory prescribed guidelines for Good Laboratory Practices (GLPs) $[23,24]$. These cover a variety of factors including requirements for employing a written study protocol, standard operating procedures for all components of the study, specifications for animal housing and welfare and procedures for record keeping and archiving. Although not all of the tests submitted under the HPV Challenge Program may meet the criteria of current test guidance, such as the older tests that were performed before development of guidelines, the design and results of such tests should be considered, and such tests at least evaluated, in order to determine the contribution of the older tests to the database for the chemical. Clearly, tests performed under guideline testing protocols will be the most relevant for current risk assessments and establishing the toxicity profile of a compound. The following is a brief discussion of the major classes of toxicity tests that are included in standard toxicity testing paradigms for industrial chemicals, including a discussion of the type of data collected, and how the data relates to humans of various ages, with a focus on children.

\section{Acute Toxicity Tests}

Acute, high dose exposure and the resulting toxicity responses represent a scenario that is highly relevant to understanding the potential consequences of short-term human exposures (e.g., intentional or accidental poisoning in adults and children). As a result, data from acute toxicity testing in animals provides important human health risk information. Acute toxicity protocols usually prescribe use of rodents, such as rats or mice, and the most appropriate route of exposure (oral, inhalation and/or dermal) as it relates to expected human use patterns. In many cases for industrial chemicals, human exposure can be through all three routes. These tests are commonly performed on post-pubertal animals of both sexes. These are single dose studies with observation periods after dosing of up to 2 weeks. Currently, performed tests (guideline-compliant tests) involve complete post-mortem exams on all survivors as well as animals that might die during the course of the study. At necropsy, all macroscopic lesions observed are examined and recorded, and target organs and cause of death are identified, whenever possible.
Animals are also examined twice daily for clinical signs and symptoms of toxicity; examples of the types of symptoms and signs observers look for are listed in Table 1. Thus, the observational data and the gross pathological examination data collected on guideline-compliant studies provide a great deal of useful information to the toxicologist concerning what organs are targeted by the test chemical and what subtle as well as overt signs of toxicity to look for in humans who could be over exposed to such substances.

Because of the nature of the exposure (single dose), these tests are most relevant for evaluating the effects likely produced due to short-term (or intermittent) exposures to chemicals. These tests typically do not employ infant or fetal animals, yet the information gathered has routinely been considered by toxicologists and regulatory agencies in the United States to be relevant to assessing risks of acute, high $\mathrm{dose}^{4}$ exposures in humans of all ages, as evidenced by the inclusion of such tests in standard toxicity screening lists for almost all chemicals under the scrutiny of regulatory agencies (i.e., FDA, USEPA, and the Consumer Product Safety Commission or CPSC). Although the acute toxicity test results are clearly relevant data, it is commonly accepted that additional data, beyond acute toxicity data in animals, are desired in order to adequately protect humans of all subgroups. By giving large doses of the chemical in a single dose, these studies are not designed to delineate small differences in response among animals but instead to delineate effects in post-pubertal animals as a guide for the identification of target organs of toxicity in humans of all ages. Although it is true that fetal and newborn animals might respond differently in the case of some chemicals, it is also true that animals with different genetic backgrounds, nutrition and environments might respond differently. For over 50 years, acute toxicity tests have provided toxicologists and health professionals with information that has been used to classify substances according to their relative degree of acute toxicity [25].

\footnotetext{
4 "High dose" in this context refers to doses that are always higher than anticipated human exposures. The only exception to this would be intentional or accidental poisoning episodes in humans.
} 
It is useful to provide examples of how acute toxicity test data are used for human health hazard delineation. For example, the acute toxicity test is used as a guide to ensure safe and effective design of packaging, transport and handling procedures for chemicals and chemical products. In addition, acute tests provide critical information for determining appropriate occupational industrial hygiene practices and personal protective equipment. In the United States, many regulatory programs, including USEPA, the Department of Transportation (DOT), the Occupational Safety and Health Administration (OSHA), the CPSC, and others require or use information from acute tests to develop the language on product labels which informs users not only of the proper procedures for using the product, but also provides information on signs and symptoms of acute health effects from inadvertent over-exposures. For example, all pesticides, including disinfectants are required by USEPA to be labeled "Keep Out Of Reach Of Children" and to have a signal word, such as "Danger", "Warning" or Caution"; the signal word is dictated by regulation and is dependent upon the degree of toxicity demonstrated in tests of acute toxicity in animals [26]. From the standpoint of children's health, acute toxicity testing results are very informative for safety assessment of products and substances that children contact. The CPSC relies on the results of toxicity tests for prescribing labeling of products, including those intended for children's use. The CPSC requirements include signal words based on toxicity determinations ("Danger", "Caution", "Warning") and precautionary/first-aid measures. Furthermore, CPSC has the authority to ban hazardous substances from products that children may access based on a determination of the degree or nature of the hazard.

\section{Repeat Dose and Subchronic Toxicity Tests}

Repeated exposure to lower levels of chemicals, levels that do not produce acute, frank toxicity, is another relevant exposure scenario for human health risk assessment. Thus, data from studies in laboratory animals are routinely collected for most chemicals in repeated dose toxicity testing.
Repeat dose and subchronic toxicity studies provide information on the major toxic effects of the test substance, indicate the target organs affected, and provide information on dose response for effects. Once identified, the dose response is used to establish acceptable daily intake levels for humans, using either No Observed Adverse Effect Levels with application of uncertainty or safety factors as discussed above, or by employing the Benchmark Dose approach [27].

Repeat dose toxicity testing can vary in length from as little as two weeks to as much as 90 days of exposure to a test chemical. Studies longer than 90 days or three months are usually termed chronic toxicity tests. Under the OECD SIDS program, a 28-day repeat dose study has often been employed. However, the current practice in the U.S. for industrial chemicals would generally be a 90-day test in rodents. For the HPV Challenge, both 28-day repeat dose studies and 90-day subchronic studies can be submitted and considered.

Subchronic studies usually employ four test groups (control, low dose, mid dose, and high dose) so that a doseresponse assessment can be performed. Both male and female animals are used in such studies, and studies are typically conducted on young adult laboratory rodents (animals that are 6 to 9 weeks of age). Similar to the acute toxicity tests, post-mortem exams are performed on all survivors of the study as well as all animals found dead or moribund. Microscopic pathological exams and clinical laboratory evaluations are routinely conducted in subchronic studies. The clinical laboratory parameters typically assessed are listed in Table 2. Histopathological examinations normally begin with the control and high dose animals, and include any animals in other test groups that died during the course of the study or developed visual lesions or pathology during the course of the test. A list of the organs and tissues usually examined as part of a subchronic toxicity study is provided in Table 3. In addition to the high dose groups, histopathological examinations of lower dose groups continue for suspected target organs and to identify no effect levels of

Table 2. Assays of Biological Fluids Conducted As Part Of Subchronic and Chronic Studies

\begin{tabular}{|c|c|c|}
\hline Hematology & Clinical Chemistry & Urinalysis \\
\hline \hline red blood cell count & alanine aminotransferase & volume \\
\hline hematocrit & aspartate aminotransferase & specific gravity \\
\hline hemoglobin & gamma-glutamyl transpeptidase & plotein \\
\hline leukocyte count & glucose & ketones \\
\hline clotting function & bilirubin & occult blood \\
\hline platelet counts & creatinine & sediment microscopy \\
\hline & urea & bilirubin \\
\hline
\end{tabular}


Table 3. Organs Routinely Assessed for Gross and Microscopic Pathology in Subchronic Toxicity Tests

\begin{tabular}{|c|c|c|}
\hline adrenals & heart & ovaries \\
\hline bone & kidney & pancreas \\
\hline bone marrow & large intestine & prostate \\
\hline brain & liver & small intestine \\
\hline esophagus & lung & bladder \\
\hline salivary glands & testes & uterus \\
\hline spleen & thymus & any target organs identified \\
\hline stomach & trachea & lymph nodes \\
\hline
\end{tabular}

exposure. Since 1998, subchronic studies conducted in accordance with the USEPA test guideline include specific assessments of neurobehavioral endpoints, thereby providing important information on the potential of substances to cause neurological effects [16]. Many times these studies include satellite groups which are treated exactly the same as the core group for the duration of the study, and then treatment is discontinued, while the animals are maintained for a further period of time to evaluate reversibility, persistence or delayed occurrence of toxic effects.

Subchronic toxicity studies identify the targets of toxic effects associated with repeated exposure to a substance. Such information provides key insights into the tissues, organ or cell systems that could be affected in humans by repeated exposure to such substances, and equally important, information about the dose (or exposure) levels associated with the type and degree of toxicity. Therefore, these studies add a great deal of scientific data to the toxicological profile of the test chemical. Greater attention has recently focused on immunotoxicity endpoints and application of these in hazard and risk characterizations for children. The subchronic test in the OECD-SIDS/HPV screening battery provides relevant information for screening for potential immunotoxicity, and when such a subchronic toxicity study indicates non-stress-related primary effects on immune parameters, such as abnormalities in spleen/spleen weight, changes in white blood cell counts, or abnormal lymph node histopathology, then additional, specifically-focused immunotoxicity testing should be considered [5].

Unlike acute toxicity tests, subchronic toxicity testing is relevant to assessing risks posed by low dose as well as high dose exposures to chemicals. Although fetal and neonatal organisms are not the focus of these studies, the qualitative results have been routinely applied to assessing potential for toxicity in infants and children as well as adults. The fact that these studies involve examination of a wide variety of organs and tissues at the microscopic level, as well as more subtle changes in clinical laboratory parameters, make subchronic toxicity testing an important component of risk assessments. Results of subchronic toxicity testing, when considered as part of a package of information on a chemical, provides data useful to predicting the types of toxicity that could potentially occur in children as well as adults. From the perspective of hazard characterization, to identify those organ systems that can be affected by a chemical exposure, subchronic test results are applicable to children, adolescents and adults. While there may be quantitative differences as a function of different lifestages, in general, qualitatively, the inherent toxicological properties of a given substance, a substance's mode of action, and the interaction with specific biological systems, does not vary from one life stage to another.

\section{Genotoxicity Testing}

Genotoxicity tests are designed to examine the effects of chemicals on the hereditary material (DNA) and the processes that control the expression of this genetic material in living cells. As a result, the data gathered from such tests are directly relevant to assessing potential risks posed by chemicals regardless of the age of the individual. This is because all cells from living organisms after birth and even during the fetal period are dependent on the same processes that control genetic or DNA expression. Genotoxicity testing programs for chemicals routinely involve a variety of tests that target different endpoints of genetic expression. Table 4 lists types of genotoxicity tests that are most often employed in industrial chemical testing. Typically, genotoxic potential is assessed by evaluating results from a battery of assays. Available assays can detect mutational activity in bacterial as well as mammalian cells, chromosomal damage both in vitro and in vivo, and DNA damage and repair capability. When a battery or collection of genotoxicity tests is performed, the results give valuable information that is applicable to the potential of the chemical to damage cells and affect DNA expression in cells from humans of all ages. Genotoxicity data are therefore directly relevant to assessing risks posed to children following exposure to a chemical.

Genetic toxicity testing is particularly informative for evaluating potential carcinogenicity. Employing a biologically-based trigger as part of a tiered testing framework, a trigger that integrates results from genetic toxicity tests with results of a subchronic study, can assist in determining whether a chronic toxicity/oncogenicity assay is warranted [5]. Since a publication in 1994 [28], greater attention has focused on quantitative assessments of cancer risks arising from early life exposures. Although it may seem counter intuitive, early life exposure does not universally lead to increased susceptibility of developing cancer. When experi- 
Table 4. Types of Genotoxicity Tests Employed in Testing of Industrial Chemicals

\begin{tabular}{|c|c|}
\hline In Vitro Tests & In Vivo Tests \\
\hline \hline bacterial mutagenicity assays & mammalian bone marrow cytogenetics (chromosomal analysis) \\
\hline mammalian bone marrow cytogenetics (chromosomal analysis) & mammalian bone marrow cytogenetics (micronucleus assay) \\
\hline mammalian bone marrow cytogenetics (micronucleus assay) & sister chromatid exchange assay \\
\hline unscheduled DNA synthesis assay & \\
\hline sister chromatid exchange & \\
\hline
\end{tabular}

mental data of repeat dose, early life exposures to chemical carcinogens were evaluated, almost $60 \%$ of the datasets indicated equal or less sensitivity of the early life exposure period compared to exposure later in life [30]. Early-life exposures to carcinogens has been shown to lead to both increased and decreased susceptibility [29, 30] and EPA has developed specific guidance for conducting quantitative risk assessments for early life exposure to chemicals that may be carcinogenic [31]. Furthermore, NTP has decided to consider, on a case by case basis, whether to initiate dosing at the customary young-adult stage, or instead, to start the experiments with prenatal dosing [32].

\section{Reproductive and Developmental Toxicity Testing}

Tests to evaluate the potential for a substance to cause toxicity during in utero development and postnatally during growth and maturation are of direct and obvious importance to characterizing health hazards to children. The evaluation of substances for their potential to adversely affect development and reproduction is founded upon the scientific understanding of ontogenesis, integral to which is the concept of windows of vulnerability both for developmental toxicity and reproductive cells and organs. Windows of vulnerability during fetal development and sexual maturation are not a new concept, as these have been incorporated into research, testing and safety assessments for more than 40 years [33]. It has been said that any agent administered at an appropriate dose and at an appropriate time of development in a sensitive species can cause some type of disturbance in development [34]. Thus, the magnitude, frequency, duration and timing of exposure play a critical role in studies to evaluate the potential for agents to adversely affect development and reproduction.

There is increasing public attention to the potential hazards to the developing fetus and to reproduction. Reproductive toxicity includes adverse effects on fertility in males and females and on developmental toxicity, and covers any effect interfering with normal development both before and after birth, from conception to sexual maturity. Reproductive and developmental toxicity testing are a standard part of the toxicity testing program for drugs, medical devices, biologics, food additives, pesticides and are also endpoints required for industrial chemicals under the HPV Challenge and OECD SIDS programs. Reproductive toxicity testing is generally focused on determination of the potential of a chemical to affect the ability of an organism to reproduce, while developmental toxicity testing focuses on the potential of a chemical to affect the viability or normal development of offspring of an organism during gestation. There are a number of standardized test methods that can be used to evaluate the effects of substances on development and reproduction $[20,21]$. Typical developmental toxicity tests evaluate the effects of exposures during organogenesis and histogenesis, those periods during which organ systems are differentiating, forming and growing in utero. In developmental tests, pregnant animals are treated with the test agent (thus exposing the offspring in utero) and then fetuses are evaluated just before parturition for effects on the skeletal and organ systems. The period that is covered by the developmental toxicity study is sensitive to induction of structural malformations (birth defects).

Reproductive tests can include one, two or more generations. The purpose of these studies is to examine successive generations to identify possible increased sensitivity to a chemical, effects on the fertility of male and female animals, prenatal, perinatal, and postnatal effects on the ovum, fetus and offspring, including teratogenic effects, as well as perinatal and postnatal effects on the mother. In such tests, the males and females of the parental generation are exposed to the test substance prior to mating. Exposure of the parental generation (males and females) continues throughout the gestation and weaning periods (offspring continue to be exposed via their mother through lactation for test agents that are transferred into milk). After weaning, the offspring are placed on a direct exposure regimen. Exposure is continued through the stages of adolescent growth and development, and at the stage of sexual maturation, in multigeneration studies, the exposed animals are mated and the effects on reproduction are evaluated.

In some cases, a screening test design is used in which an assessment of developmental and reproductive toxicity is combined into a single study design (see TG 421 and TG 422 in reference 22). In this assay, effects on reproduction of males and females as well as effects upon development of the offspring are examined. In multigeneration studies, exposure is continued during the time of organogenesis of the ovaries and testis in order to investigate whether a chemical causes increasing toxicity and reproductive problems during subsequent generations. At weaning, the exposure is continued, and the effects upon reproduction are evaluated. Because reproductive and developmental toxicity studies have not been designed in the past to evaluate systemic toxicity in animals less than 3-4 weeks of age, recent guidelines have attempted to address this issue by recommending that target organs, if known or identified, be evaluated histopathologi- 
cally in the F1 generation of reproductive studies. Table 5 lists endpoints that are examined in a standard multi-generation reproductive and developmental toxicity test, endpoints that cover all periods of animal development from conception, through the fetal period, and after birth into newborns (infants).

Reproductive and developmental toxicity tests are directly applicable to assessing risks posed by chemical exposures in fetuses, infants and children as the animals in the tests are exposed to the test chemical as they are conceived, through in utero development, and during infant development. These studies provide information relevant to assessing the potential of a substance to cause birth defects or harm to growth, development, maturation and reproduction - all of which are important considerations in assessing the safety of chemicals to which children may be exposed. For example, results of these studies are used by manufacturers and formulators in developing the Material Safety Data Sheets which accompany all chemical products and which include a description of the toxicity hazards. Further, OSHA relies on these types of toxicity studies to direct appropriate occupational hygiene measures to protect workers from adverse reproductive effects, and to protect against teratogenesis (29 CFR Part 1910.1200, Pages 479 - 481, Hazard Communication).

Research has been performed to assess the adequacy of standard testing protocols to identify reproductive and development hazards. In two review papers, Claudio and colleagues assessed the adequacy of USEPA test methods for identification of hazards to developing organisms $[35,36]$. In the case of both the reproductive and developmental toxicity guidelines, the authors identified certain limitations in the testing protocols. In particular the authors suggested that the developmental toxicity study protocol did not allow for exposure of animals during all possible predicted human scenarios while some of the reproductive protocols did not examine the paternal contribution to offspring toxicity. However, the authors concluded that the data collected, despite the limitations, were relevant to assessing risks to children's health. In an earlier assessment focused on risk of developmental toxicity (teratogenicity), the concordance of animal and human data was reviewed for 38 drugs reported to be developmental toxicants in humans, and 165 drugs reported not to produce developmental toxicity [37]. The results of the assessment showed that of 38 drugs identified as human teratogens, 37 were positive in at least one animal species, and 29 were positive in more than one species. Further, among 165 compounds identified as being "nonteratogenic" in humans, only 47 were negative in all animal species tested. These data indicated that testing performed in animals is relevant for assessing human teratogenic hazards. In a statistical analysis of the predictive power of developmental toxicity testing in animals, the results suggested that animal data are useful for identifying human developmental toxicants, although not always determinative [38]. In the recent review of the issue of relevancy of animal testing for assessing potential hazards to children, a panel of scientists working on behalf of WHO concluded that toxicity testing in experimental animals plays a key role in identifying and characterizing hazards for children and that, further, most chemicals known to cause developmental effects in humans have been identified as such through data in at least one animal species [3]. Therefore, even with the complexity of human development and known differences between species in development, a variety of analyses have suggested that animal toxicity testing data are adequate for predicting developmental toxicity potential of chemicals.

Table 5. Endpoints Assessed in Standard Multi-Generation Reproductive and Developmental Toxicity Studies

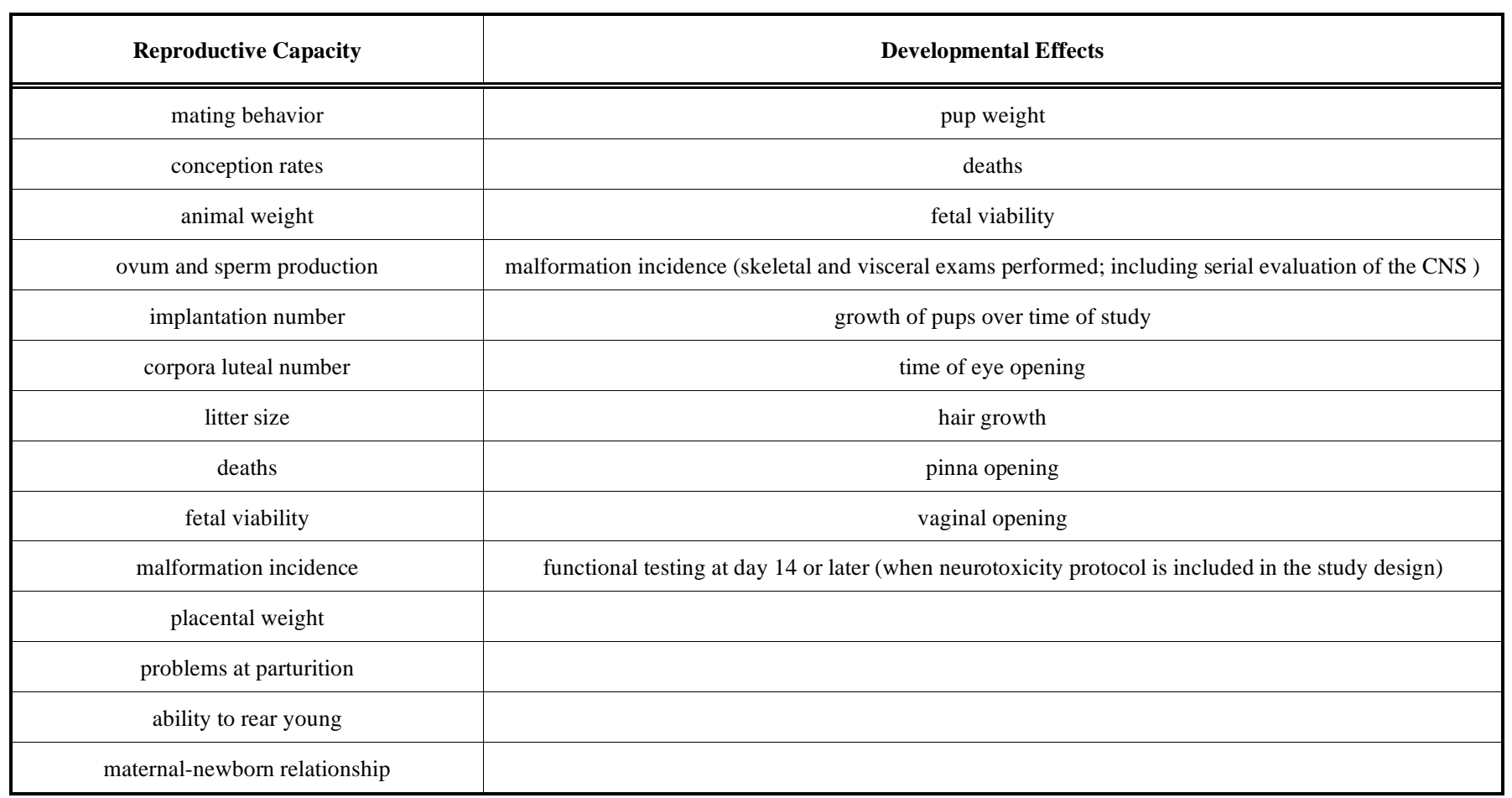


Although there are cases where the developing organism has been shown to be more sensitive to certain exposures than an adult, this is not a universal axiom. It is true that children are not "little adults" in terms of their responses to chemicals and their exposure to chemicals [39]. That does not imply, however, that standard toxicity data, gathered through standard toxicity testing protocols, is irrelevant to assessing risks in children. Age is only one of the factors that can lead to susceptibility and in some cases may not be the most important factor. The scientific literature shows that children may be more sensitive, equally sensitive or less sensitive to effects from chemical exposures than adults [39]. While children over 6 months of age can be more sensitive than adults to chemical toxicity, in many cases they are less sensitive [40]. Furthermore, Dourson et al. [41] concluded that, taking into account relative sensitivities of adults and children and the types and the scope of toxicity test protocols that substances are subjected to, additional uncertainty factors, over and above those traditionally used, are generally not supported for addressing concerns to children greater than 6 months of age.

\section{Neurotoxicity Endpoints}

When children are a population of concern in a human health risk assessment, data on the potential neurotoxic effects of chemicals are particularly useful since the brain is undergoing critical development during gestation, infancy and early childhood. Every one of the in vivo mammalian toxicity tests conducted as part of the OECD-SIDS battery the acute study, the subchronic study, the developmental toxicity study and the reproduction study - includes some degree of assessment for neurotoxicity. Gross and microscopic evaluations of abnormalities of the nervous system are routine components of these protocols. Assessment of neurotoxicological signs such as seizures, narcosis, and neuromotor dysfunction, are included as requirements in each of these toxicity tests. Formal observations of the laboratory animals are generally conducted twice each day during the course of toxicity studies, including specific examinations for signs of neurotoxicity, signs such as changes in gait, posture, movements, and stereotypic or unusual behaviors. For the most part, in acute studies, evaluation of neurotoxicity is based largely on clinical observations by trained laboratory animal technicians. However, neurotoxic effects on complex integrative functions such as motor performance, sensory acuity, memory and cognitive effects can only be quantified by specific studies. Although neurotoxicity tests were not specifically included in OECD SIDS at its inception, it is increasingly common to include a functional observational battery (FOB) in the design of a subchronic study. In fact, the OECD and USEPA now require a FOB as part of the test guidelines for both 28-day repeat dose and 90-day subchronic studies, respectively $[16,22,42]$. The FOB requirement went into effect in 1995 and in 1998, for the OECD 28day repeat dose study and the EPA 90-day subchronic study, respectively. New studies, undertaken since these dates, would include the FOB. The FOB is a series of tests specifically designed to examine the potential effects of a compound on neuromotor skills, sensory perception, and learning/memory. If the results of one or more of the OECD SIDS toxicity tests indicate particular concern for neurotoxicty, then, utilizing a tiered testing approach, more specialized studies can be triggered, as described by Becker et al. [5]. Thus, the OECD SIDS battery includes toxicity assays that can detect the neurotoxic potential of chemicals, and this is an important component used in safety evaluations for both adults and children.

\section{HPV Chemical Challenge Program: Analysis of Avail- able Data}

In the US HPV Challenge Program, more than 300 companies and consortia agreed to complete the OECD SIDS battery for approximately 2200 chemicals, and make the results publicly available. Toxicity study results gathered in the types of tests summarized in the preceding section, which as discussed are considered relevant to assessing potential hazards to humans, including children, are included among the many other types of information developed for this program. Although the full complement of studies has not been reported for all chemicals, as of September 2007, information on 1399 substances have been made publicly available on the USEPA website http://www.epa.gov/hpv/. The initial task of the HPV sponsors was to collect existing relevant toxicity studies, to evaluate their adequacy to satisfy the OECD SIDS toxicity battery, to develop and publicly post "test plans" which describe the available information, and to set out recommendations for which specific additional tests might be necessary to assure a complete test battery. Information was gathered for both single chemicals and chemicals representing a "category." For the HPV Challenge program, the USEPA defines a chemical category as "a group of chemicals whose physicochemical and toxicological properties are likely to be similar or follow a regular pattern as a result of structural similarity." ${ }^{\circ}$ After taking into consideration comments on these "test plans," sponsors then conduct any specific additional studies deemed appropriate and report results. Although there was speculation that the HPV Challenge might require considerable testing, early responses indicated that, for both specific chemicals and categories of chemicals, a significant amount of toxicity data and endpoints of concern had been previously generated, but had not been formally submitted to EPA because there was no requirement to do so. An analysis of submissions made in the early period of the HPV Challenge (February 2003; data not shown) revealed 984 chemicals covered by submissions as of that date. The percentage of chemicals with information deemed by the sponsors to be sufficient to fulfill the endpoints ranged from $88 \%$ for acute toxicity, $72-74 \%$ for genotoxicity, $80 \%$ for repeat dose toxicity and $68 \%$ for reproduction and development endpoints (combined) (data not shown). Importantly, from the perspective of potential hazards to children, even at that point in time, for approximately 1000 chemicals, more than 600 robust summaries ${ }^{6}$ of studies on reproductive toxicity, developmental toxicity or the combined endpoints were submitted.

\footnotetext{
${ }^{5}$ http://www.epa.gov/hpv/pubs/general/categuid.htm

${ }^{6}$ Robust study summaries were prepared in accordance with EPA guidance (http:// www.epa.gov/hpv/pubs/general/robsumgd.htm). Robust summaries are"... intended to provide sufficient information to allow a technically qualified person to make an independent assessment of a given study report without having to go back to the full study report, and to also allow evaluation of the proposed test plan. A robust study summary therefore reflects the objectives, methods, results, and conclusions of the full study report, which can either be an experiment or in some cases an estimation or prediction method."
} 
Subsequently, more chemical information has been submitted and where additional studies were needed to provide hazard information for specific chemicals, studies were conducted and those data were submitted as well. As of August 2007, USEPA has posted information covering a total of 1,399 HPV Challenge chemicals. The data in Fig. (1) indicate those studies, broken out by endpoint, for which there are available data or information that the sponsors have deemed sufficient to satisfy the specific OECD SIDS study type. With respect to study endpoints, the percentage of chemicals with sufficient information ranged from $92.4 \%$ for reproduction and developmental (combined) toxicity studies to $98.6 \%$ for acute toxicity studies The studies already communicated by the sponsors include more than 1,200 specific toxicity studies on reproductive toxicity, developmental toxicity (or the combined endpoints); because of the category approach, the sponsors can apply the information of the results of these studies to cover, by inference, chemicals with demonstrated similarities. Specific guidance has been provided by EPA for developing categories (http://www.epa. gov/hpv/pubs/general/categuid.htm), which includes consideration of functional groups, common precursors, breakdown products, metabolism and metabolites, and many other factors. Because of recognized limitations of inference ap-
Although the HPV Challenge is behind the target dates established when the initiative was first started, the program's value is clearly shown by the breadth and depth of the toxicity data provided, and, as a result, the program has been recently extended and expanded by industry [43] for substances that have become HPVs in the period since the HPV Challenge started.

EPA has recently published screening-level hazard characterizations for over 200 high production volume chemicals. ${ }^{7}$ Hazard characterizations are prepared from EPA's scientific review of the screening-level hazard data set contained within each HPV Challenge Program submission. Agency reviews are performed according to established HPV Challenge Program guidelines and USEPA risk assessment guidance.

With the availability of screening-level hazard characterizations, we performed an analysis of the data that was submitted to EPA with the goal of determining whether the data had relevancy to children and characterization of children's risk. In our analysis, summarized in Table $\mathbf{6}$, the types of data considered of most immediate relevancy to characterization of the potential risks to children included reproductive toxicity data, developmental toxicity data and

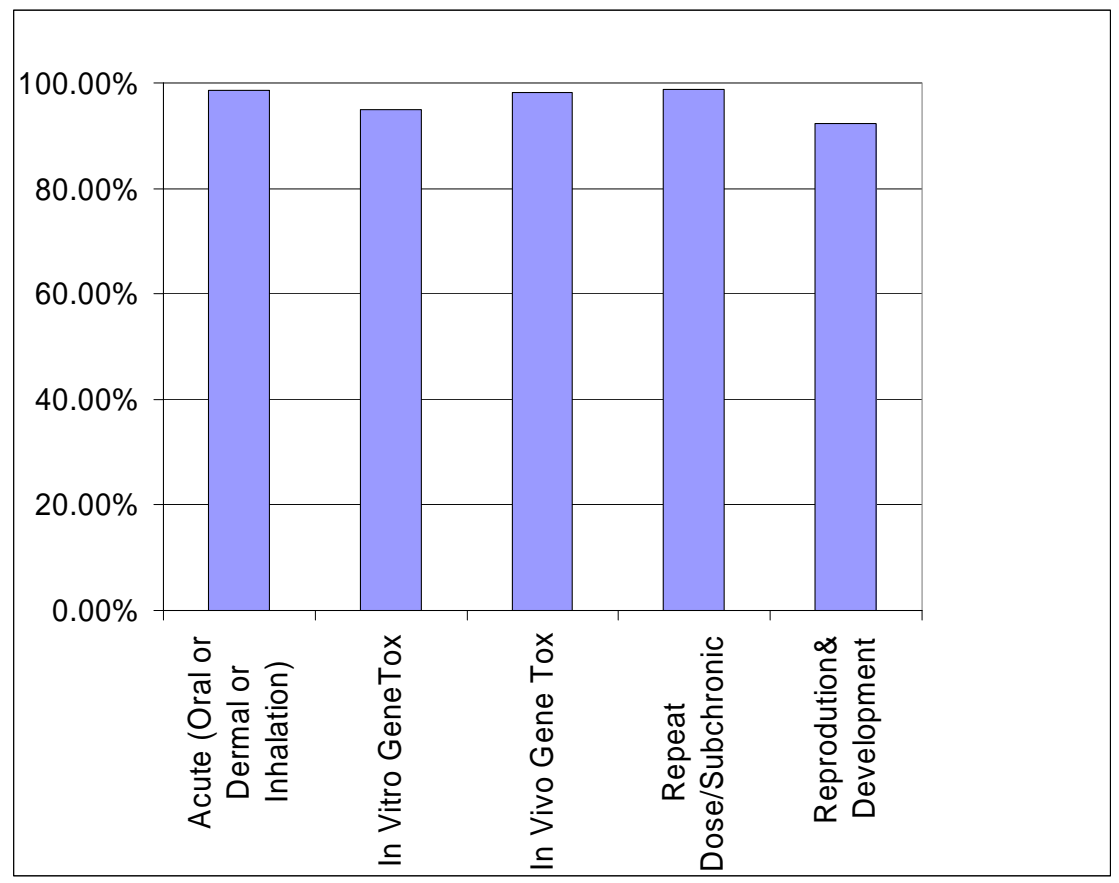

Fig. (1). Percentage of Submitted HPV Chemicals (Including Those Grouped Into Categories) with Fully Characterized Hazard Information for Each Test/Endpoint.

The current results of the HPV Challenge Program are summarized. As of August 1, 2007, the USEPA had posted information covering 1,399 chemicals in the HPV Challenge Program (both single chemicals and chemicals representing a "category"). The percentages indicate the percentage of studies within each endpoint for which the Sponsors' have determined the data (both existing and newly generated) are sufficient to satisfy the study protocol and data requirements.

proaches to toxicity characterization, the HPV Challenge requires that once the testing within a category is completed, an analysis is to be conducted to support or refute the proposed category. If supported, there's confidence that the available test data can be used with reasonable certainty for interpolation or extrapolation to other substances that comprise the specific category. genotoxicity data, as all three types of data either involve exposure of developing organisms or relate to the potential

\footnotetext{
${ }^{7}$ Of the screening assessments posted by EPA as of May, 2008, approximately 70 are for individual chemicals while approximately 30 represent groups of highly related chemicals or chemical mixtures that together represent a total of approximately 200 substances. http://www.epa.gov/hpvis/abouthc.htm
} 
to affect growth and development of cells, organs, and the whole organism. In the case of the chemicals included in the HPV program, such toxicity data were available in almost all cases. If such data had not been available, our analysis would have extended examination to subchronic, repeat dose and acute toxicity studies. As shown in Table 6, the data in these hazard characterizations are clearly useful for screening potential hazards to children as well as adults because they included methodical consideration of data from reproductive toxicity, developmental toxicity and genotoxicity studies, the studies of most relevance for assessing effects from prenatal exposures, post-natal exposures on growth, development and maturation. Therefore, by coupling child-specific exposure data/information with the hazard data from the HPV Challenge, child-focused screening level risk evaluations would be possible.

\section{Can Standard Toxicity Tests be Used to Screen Indus- trial Chemicals for Potential Hazards to Children's Health? - The HPV Challenge Program as a Case Study}

Government laboratories and program offices as well as industry have long engaged in product and chemical safety testing and risk assessments. Over the years, these procedures have become more harmonized, and standardized toxicity testing protocols and test batteries now comprise the scientific foundation of such assessments. While there are different types of evaluation systems for existing industrial chemicals, OECD SIDS represents a valuable, proven and appropriate battery for conducting initial hazard assessments for HPV chemicals, and for determining testing priorities (whether additional testing is warranted and which endpoints need further elucidation). While the OECD SIDS/HPV Challenge battery does not include every possible toxicity test, the mammalian toxicity tests included in the battery provide valuable insight into the potential hazards to humans, including children. Because of the study designs and breadth of these investigations, data and information developed under the OECD SIDS program and the HPV Challenge Program can be considered relevant for evaluation of the potential health hazards to children when information exist indicating exposure to children is a concern. Further, the results of the analysis above demonstrate that the assertion that there is little relevant information for characterizing children's health hazards for HPV chemicals is simply not borne out by the evidence we have today. There are in fact large amounts of data that have been collected specifically on the types of studies that have information relevant to children's hazard identification, in particular information from studies conducted in pregnant animals and animals exposed in utero and immediately after birth.

One question that can be raised regarding the present analysis is whether the current protocols for assessing reproductive and developmental toxicity in animals are missing some critical endpoint that limits their utility for hazard characterization in children. This question was a central issue in a recent paper by Morford and colleagues [44]. In that paper, the authors examined the predictability of children's health risk from animal data, considering the current standard protocols for reproductive and developmental toxicity tests. They concluded that current toxicity testing protocols for evaluating growth, survival, and morphological changes due to exposure of humans during gestation (in utero) are adequate for assessing children's health risk. The authors did, however, indicate that additional emphasis should be placed on developing new testing methods to examine the full impact of postnatal exposures to chemicals. Thus, if the issue is predicting the potential for specific toxicity endpoints following postnatal exposures, the standard testing protocols currently used by all regulatory agencies, including the OCED SIDS program, could benefit from re-examination. However, for hazard characterization purposes, it remains to be demonstrated that the dose levels necessary to cause adverse effects would be lower if new postnatal endpoints were evaluated, in comparison to the dose levels producing effects with the existing protocol. In this regard, an analysis was performed of three different rat multi-generation studies conducted on one substance at different dates (1965, 1984 and 1990). The analysis allowed comparison of the results gathered using different protocols against data from an enhanced one-generation protocol that used additional postnatal animals. The analysis showed that the 1984 and 1990 multi-generation assays yielded comparable LOAELs and NOAELs for hazard characterization as did the one-generation enhanced postnatal animals/endpoint study [45].

Based upon experience with the OECD SIDS program since the mid 1980's, the evaluation and analysis conducted in this article and previous published work [5] there is reasonable justification to conclude that the current tiered testing paradigm for industrial chemicals, which commences with a base set of hazard screening information that includes tests for developmental and reproductive toxicity, appears to function adequately to screen for possible health hazards to children, as well as adults. As discussed in a previous publication [5], a tiered testing framework with toxicity triggers to assist in decision-making focuses resources on those substances that warrant greatest attention by identifying and directing efforts to those specific, higher tiered toxicity tests that are the most important for characterizing potential hazards. In addition, animal welfare concerns pertaining to refinement of toxicity testing practices and reduction of laboratory animals are advanced with such a tiered testing paradigm. However, with this paradigm, while both "false positives" and "false negatives" would be expected to have social and economic effects, "false negatives" would be of considerably greater concern from a human health perspective. Therefore, it is important that such screening level information be used within a weight of evidence framework that assigns appropriate levels of scientific confidence based on the strengths and limitations of each study, both design and results, and the overall dataset for a given chemical. When information is sparse, health protective interpretations and assumptions are appropriate, such as was discussed above regarding use of additional uncertainty factors when datasets are incomplete or of low confidence. In addition, for hazard characterizations for children based on limited datasets, "signals" from lab animal studies of developmental and or reproductive toxicity should clearly be given weight when assigning hazard designations. With such approaches for interpreting and applying the data in decision-making, the likelihood of a "false negative" should be greatly reduced, although it can never be eliminated all together. As indicated in Table 6, EPA has approached hazard characterizations for HPV substances with due concern, as approximately $90 \%$ of the entries designated as "high" concern have datasets with 
Table 6. Results of the EPA Screening Level Hazard Assessments for HPV Chemicals as Posted May 2008: Data Showing "Toxicity Tests Relevant to Evaluating Potential Hazards to Children",1

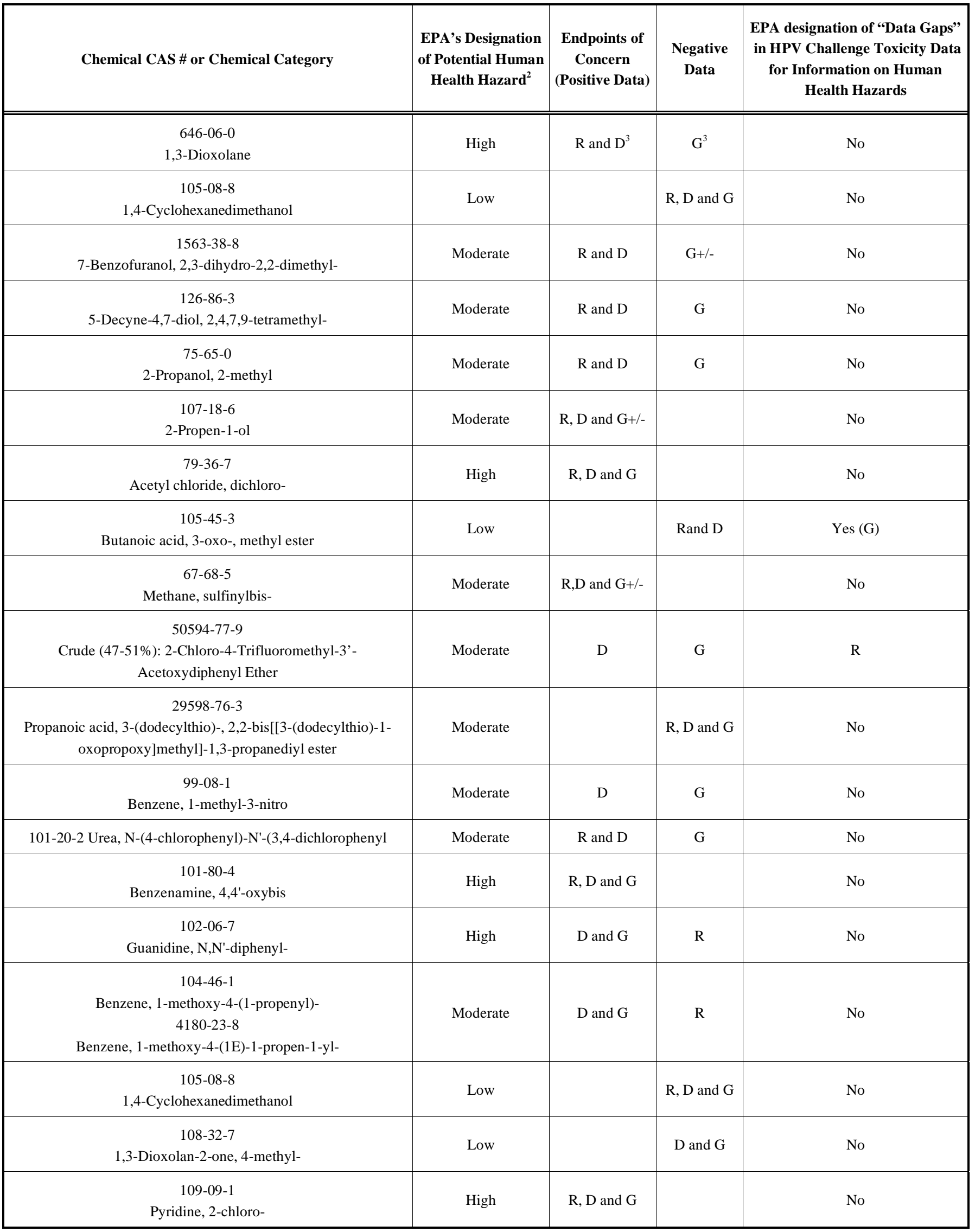


(Table 6) contd...

\begin{tabular}{|c|c|c|c|c|}
\hline $\begin{array}{c}110-71-4 \\
\text { Ethane, } 1,2 \text {-dimethoxy- }\end{array}$ & High & $\mathrm{R}, \mathrm{D}$ and $\mathrm{G}$ & & No \\
\hline $\begin{array}{c}111-96-6 \\
\text { Ethane,1-1'-oxybis[2-methoxy- }\end{array}$ & High & $\mathrm{R}$ and $\mathrm{D}$ & G & No \\
\hline $\begin{array}{c}115-10-6 \\
\text { Methane, oxybis- }\end{array}$ & Moderate & $\mathrm{D}$ & G & No \\
\hline $\begin{array}{l}1222-05-5 \\
\begin{array}{l}\text { Cyclopenta[g]-2-benzopyran, } 1,3,4,6,7,8 \text {-hexahydr o- } 4,6,6,7,8,8- \\
\text { hexamethyl- }\end{array}\end{array}$ & Moderate & $\mathrm{D}$ & $\mathrm{R}$ and $\mathrm{G}$ & No \\
\hline $\begin{array}{l}\text { 123-39-7 } \\
\text { Formamide, N-methyl- }\end{array}$ & High & $\mathrm{R}$ and $\mathrm{D}$ & G & No \\
\hline $\begin{array}{c}126-86-3 \\
2,4,7,9-T e t r a m e t h y l-5-d e c y n e-4,7-d i o l\end{array}$ & Moderate & $\mathrm{R}$ and $\mathrm{D}$ & G & No \\
\hline $\begin{array}{c}13752-51-7 \\
\text { Morpholine, 4-[(4-morpholinylthio)thioxomethyl]- }\end{array}$ & Moderate & G & $\mathrm{R}$ and $\mathrm{D}$ & No \\
\hline $\begin{array}{c}1847-58-1 \\
\text { Acetic acid, sulfo-, } 1 \text {-dodecyl ester, sodium salt }\end{array}$ & Low & & $\mathrm{R}, \mathrm{D}$ and $\mathrm{G}$ & No \\
\hline $\begin{array}{c}\text { 18760-44-6 } \\
\text { Thiophene, 3-(decyloxy)tetrahydro-, 1,1-dioxide }\end{array}$ & Low & & $\mathrm{R}, \mathrm{D}$ and $\mathrm{G}$ & No \\
\hline $\begin{array}{c}\text { 19248-13-6 } \\
\text { 1,2-Ethanediamine, N-ethyl-N-(3-methylphenyl)- }\end{array}$ & Moderate & $\mathrm{R}$ and $\mathrm{D}$ & G & No \\
\hline $\begin{array}{l}\text { 220352-35-2 } \\
\text { Phenol, tert-Butyl derivatives., phosphates (3:1) }\end{array}$ & Moderate & & $\mathrm{R}, \mathrm{D}$ and $\mathrm{G}$ & No \\
\hline $\begin{array}{c}2426-08-6 \\
\text { Oxirane, (butoxymethyl)- }\end{array}$ & High & $\mathrm{D}$ and $\mathrm{G}$ & $\mathrm{R}$ & No \\
\hline $\begin{array}{c}2781-11-5 \\
\text { Phosphonic acid, [[bis(2-hydroxyethyl)amino]methyl]-, diethyl } \\
\text { ester }\end{array}$ & Low & & $\mathrm{R}$ and $\mathrm{D}$ & No \\
\hline $\begin{array}{l}\text { 29598-76-3 } \\
\text { Propanoic acid, 3-(dodecylthio)-, 2,2-bis[[3-(dodecylthio)-1- } \\
\text { oxopropoxy]methyl]-1,3-propanediyl ester }\end{array}$ & Moderate & & $\mathrm{R}, \mathrm{D}$ and $\mathrm{G}$ & No \\
\hline $\begin{array}{c}306-83-2 \\
\text { Ethane, 2,2-dichloro-1,1,1 -trifluoro- }\end{array}$ & High & G & $\mathrm{R}$ and $\mathrm{D}$ & No \\
\hline
\end{tabular}




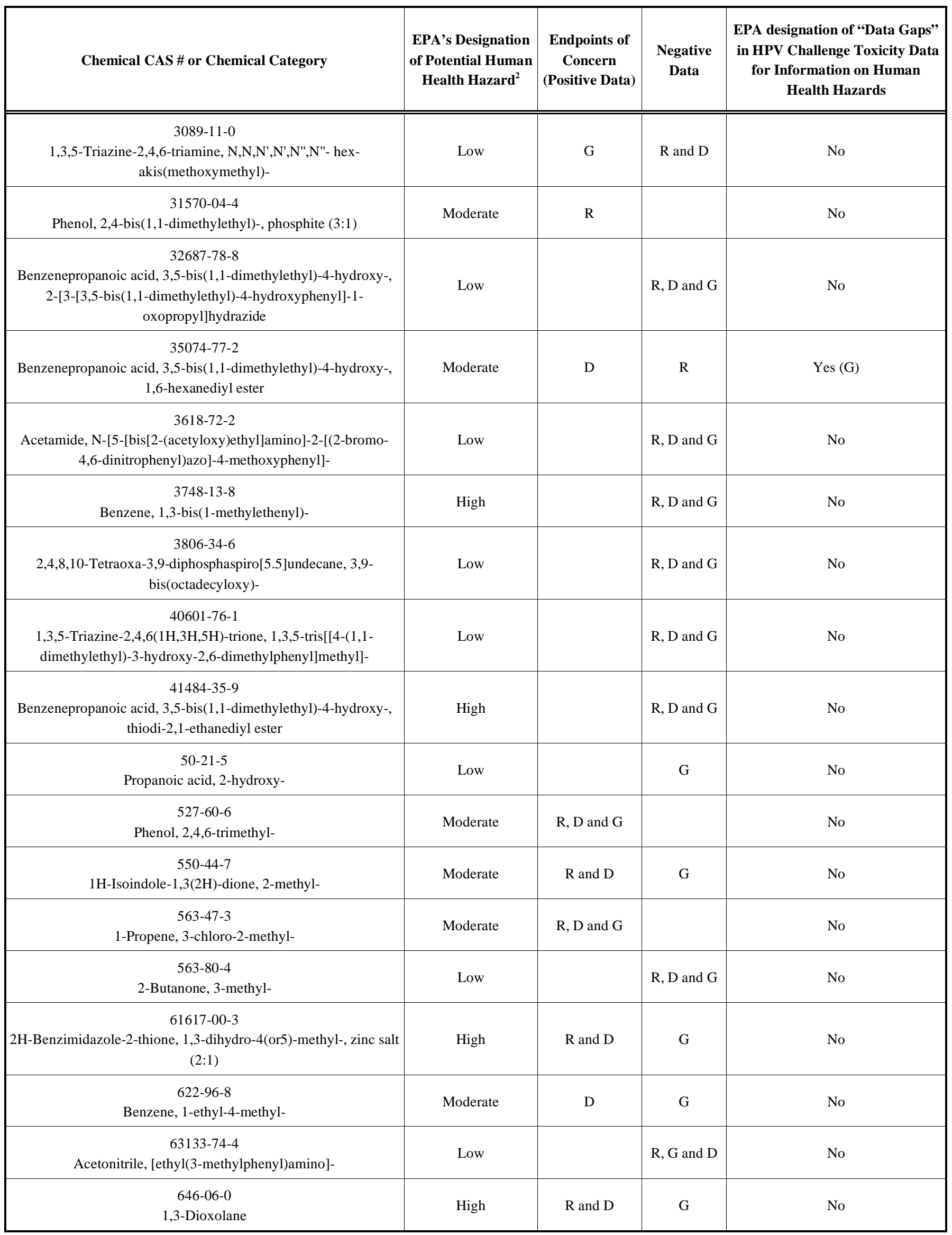


(Table 6) contd...

\begin{tabular}{|c|c|c|c|c|}
\hline $\begin{array}{c}\text { 6683-19-8 } \\
\text { Benzenepropanoic acid, 3,5-bis(1,1-dimethylethyl)-4-hydroxy-, } \\
\text { 2,2-bis[[3-[3,5-bis(1,1-dimethylethyl)-4-hydroxyphenyl]-1- } \\
\text { oxopropoxy]methyl]-1,3-propanediyl ester }\end{array}$ & Moderate & $\mathrm{D}$ & Rand G & No \\
\hline $\begin{array}{l}\text { 68227-46-3 } \\
\text { Propanoic acid, 2-hydroxy-, compd. with 2-ethylhexyl [[3-[[2- } \\
\text { (dimethylamino)ethoxy]carbonyl]amino]-4- } \\
\text { methylphenyl]carbamate }(1: 1)\end{array}$ & High & $\mathrm{R}$ and $\mathrm{D}$ & G & No \\
\hline $\begin{array}{l}\text { 75-75-2 } \\
\text { Methanesulfonic acid }\end{array}$ & Moderate & & $\mathrm{R}, \mathrm{D}$ and $\mathrm{G}$ & No \\
\hline $\begin{array}{c}\text { 8007-24-7 } \\
\text { Cashew, nutshell liquid }\end{array}$ & Low & & $\mathrm{R}, \mathrm{D}$ and $\mathrm{G}$ & No \\
\hline $\begin{array}{c}\text { 99-08-1 } \\
\text { Benzene, 1-methyl-3-nitro- }\end{array}$ & Moderate & $\mathrm{D}$ & G & No \\
\hline $\begin{array}{c}\text { 50594-77-9 } \\
\text { Phenol, 3-[2-chloro-4-(trifluoromethyl)phenoxy]-, acetate }\end{array}$ & Moderate & $\mathrm{D}$ & $\mathrm{R}$ and $\mathrm{G}$ & Yes ( $R$ and $D)$ \\
\hline Dibasic Esters Category ${ }^{4}(4)$ & Low & $\mathrm{R}$ and $\mathrm{D}$ & $\mathrm{G}$ & No \\
\hline Dicarboxylic Acids Category ${ }^{4}(3)$ & Low & & $\mathrm{R}, \mathrm{D}$ and $\mathrm{G}$ & No \\
\hline Neoacids C5-C28 Category ${ }^{4}(6)$ & Moderate & $\mathrm{R}$ and $\mathrm{D}$ & G & $\begin{array}{l}\text { Yes ( } \mathrm{R} \text { and } \mathrm{D} \text { for } \\
\text { some category members) }\end{array}$ \\
\hline Phenolic Benzotriazoles Category ${ }^{4}(4)$ & Moderate & $\mathrm{R}$ and $\mathrm{D}$ & G & No \\
\hline${\text { Alkyl Nitriles Category }{ }^{4}(3)}$ & High & $\mathrm{D}$ & $\mathrm{R}$ and $\mathrm{G}$ & No \\
\hline Alkyl Acetate C6-C13 Category ${ }^{4}(6)$ & Low & & $\mathrm{R}, \mathrm{D}$ and $\mathrm{G}$ & No \\
\hline Mononitroanilines Category $^{4}(2)$ & High & $\mathrm{R}, \mathrm{D}$ and $\mathrm{G}$ & & No \\
\hline Terpenoid Primary Alcohols and Related Esters ${ }^{4}(4)$ & Moderate & $\mathrm{R}$ and $\mathrm{D}$ & $\mathrm{G}$ & No \\
\hline Rosin Adducts and Adduct Salts Category ${ }^{4}(6)$ & Moderate & & $\mathrm{R}, \mathrm{D}$ and $\mathrm{G}$ & No \\
\hline Rosin Esters Category $^{4}(7)$ & Moderate & $\mathrm{R}$ and $\mathrm{D}$ & $\mathrm{G}$ & No \\
\hline Final Submission for Rosin and Rosin Salts ${ }^{4}(6)$ & Low & & $\mathrm{R}$ and $\mathrm{D}$ & Yes $(G)$ \\
\hline Sulfosuccinates Category $^{4}(3)$ & Moderate & $\mathrm{R}$ and $\mathrm{D}$ & G & No \\
\hline $\begin{array}{l}\text { Final Submission for Tall Oil Fatty Acids and Related Sub- } \\
\qquad \operatorname{stances}^{4}(8)\end{array}$ & Low & Rand D & G & No \\
\hline
\end{tabular}


(Table 6) contd....

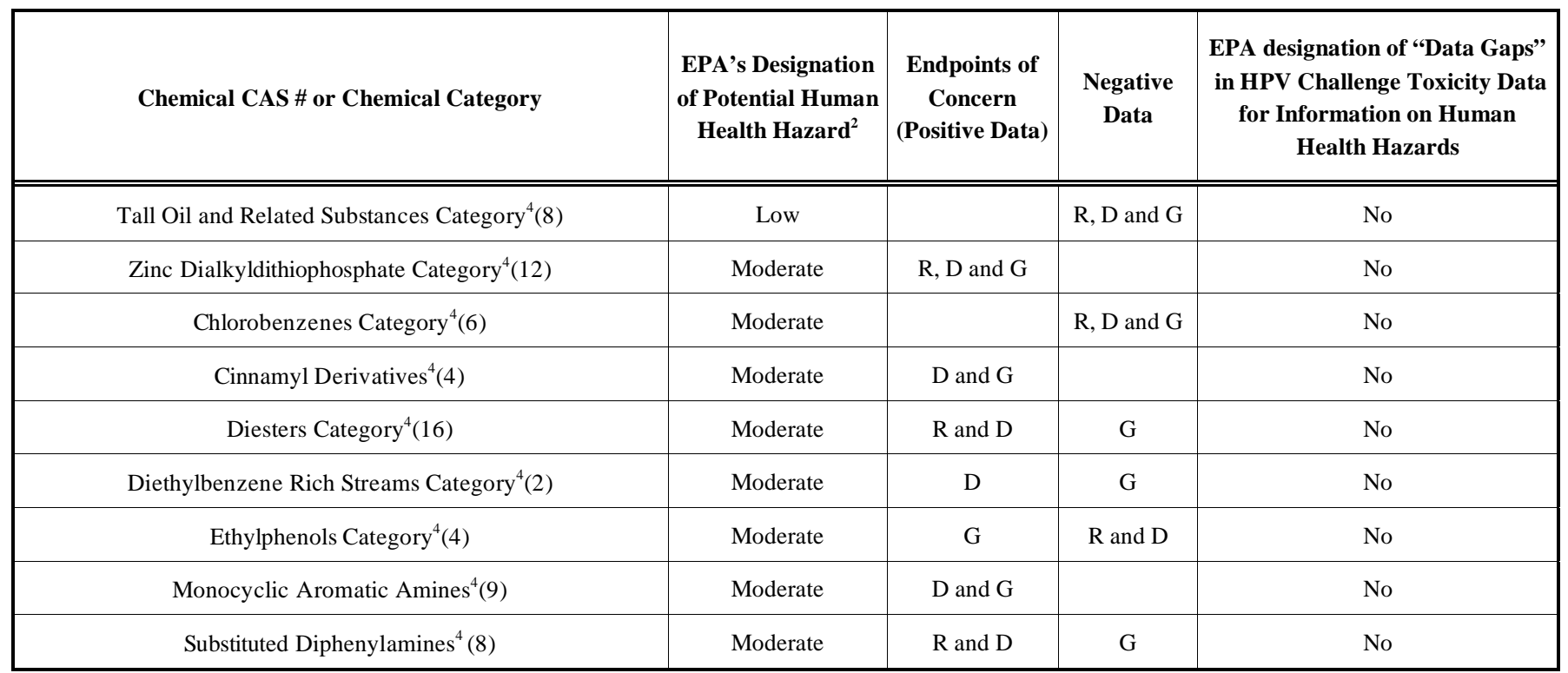

"Toxicity Tests Relevant to Evaluating Potential Hazards to Children" is defined here as the finding by EPA that the chemical or chemical group demonstrated reproductive and/or developmental toxicity in animal testing.

EPA's Designation of Potential Human Health Hazard is taken directly from the published EPA documents.

$\mathrm{R}=$ reproductive data; $\mathrm{D}=$ developmental data; $\mathrm{G}=$ genotoxicity data

Number in parenthesis indicates the number of substances contained in this category. For CAS numbers of substances within each category see EPA HPV Chemical Hazard Characterizations at http://iaspub.epa.gov/oppthpv/hpv_hc_characterization.get_report?doctype=2.

NOTE: HPV substances that were included in EPA's web postings, but for which EPA was unable or did not provide qualitative designations of potential human hazards (as of May 2008) Cas\#1333-39-7 Benzenesulfonic acid, hydroxy-; CAS\# 3757-76-4 Phenol, 2,4-dichloro-, sodium salt; CAS\#5419-55-6 Boric acid (H3BO3), tris(1-methylethyl) ester; CAS\#64667-33-0 Hexanoic acid, 4,6,6,6-tetrachloro-3,3-dimethyl-, methyl ester; CAS\#66071-92-9 Sulfite liquors and Cooking liquors, spent; Aluminum Alkyls Category; n-Butyric Acid/Anhydride Category; Triphenylboron Category; CAS\#144-35-4 2,4,8,10-Tetraoxa-3,9-diphosphaspiro[5.5]undecane, 3,9-diphenoxy; CAS\#2163-42-0 1,3-Propanediol, 2methyl-.

positive developmental and/or reproductive effects; approximately $75 \%$ of the substances judged to have "moderate" concern have datasets with positive developmental and/or reproductive effects; and only approximately $5 \%$ of the substances judged to have "low" concern have datasets with positive developmental and/or reproductive effects.

Further analysis related to concerns of the adequacy of current testing protocols to define risks to children is found in the recent series of publications that resulted from a workshop held in 2003 and focused on development of a framework for assessing risks to children posed by exposure to environmental agents [46-49]. The workshop led to the development of the USEPA's Framework for Assessing Health Risks of Environmental Exposures to Children [50]. As a result, USEPA and others now propose use of a lifestagespecific approach to risk assessment where hazard identification, exposure assessment and dose-response assessment are performed based on consideration of the most sensitive lifestage. As discussed by USEPA and others, in some cases the most sensitive lifestage may be the prenatal or immediately postnatal period, but in other cases there may be no differences in toxicity response seen regardless of lifestage; instead, it may be exposure and not toxicity response that drives the lifestage-specific analysis. What is clear from all of the recent discussion on this issue is that risk assessment methods should consider lifestage-specific analysis in order to most accurately assess risks to children's health. Collection of toxicity data generally involves testing using young adult animals for acute, repeat dose and in vivo genotoxicity assays and pregnant animals (and developing organisms) for developmental and reproductive toxicity testing. While ontogenesis may influence expression of target organ toxicity such that organotropism of toxicity in older animals could differ from that of immature, developing animals, by including acute, repeat dose, developmental and reproductive toxicity in the OECD SIDS battery of toxicity tests, such lifestage related effects, if any, would be expected to be detected.

While discussion by some have indicated potential concerns about the ability of standard toxicity tests to address those life stages most critical to assessing potential risks to children, it should not be presumed that standard testing methods are inadequate. As shown by Daston and colleagues [42], current standard subchronic, chronic, reproductive, developmental, and genetic toxicity testing protocols, when combined for any one chemical, provide data covering all parts of the human lifespan from preconception (reproduction and fertility studies) through in utero development (developmental and reproductive toxicity studies), neo-natal growth and development (reproductive toxicity study) and through old age, (chronic toxicity study). Furthermore, coverage of the life stages most critical to children's health is provided by tiered testing frameworks, such as the one described by Becker et al. [5] by beginning with the HPV Challenge toxicity studies/endpoints, and then using information gained from these specific toxicity studies to evaluate whether there is a need for, and which additional, specific type or types of toxicity tests in order to provide greater certainty of potential hazards for specific life stages or endpoints. Such tiered testing approaches can lead to a reduction 
in the number of laboratory animals used in testing without diminishing the degree of scientific certainty necessary for hazard evaluations.

The unanswered question based on current standard toxicity testing protocols that would be of most concern to children's hazard characterization may be the subtle changes that may be produced in humans but missed in animal testing because of the nature of test designs, which are not continuous from preconception through old age. However, even if current protocols lack some sensitivity for assessing certain endpoints, the standard risk assessment methods that dictate use of uncertainty factors of at least 10-fold when characterizing an exposure level as "safe" would provide a level of protection that should account for any inadequacies in testing protocols in terms of evaluation of all relevant endpoints. In fact, the use of the extra 10-fold safety factor when data relevant to assessing risks to children's health are lacking, which was initially used as a standard risk assessment practice as a "data-base uncertainty factor" and then later incorporated as part of the 1996 FQPA assessment methodology [2], is one way that risk assessment has adapted procedures to address the issue of potential increased sensitivity of children. The use of this extra 10-fold uncertainty factor, combined with the standard uncertainty factors applied when animal data are used to characterize hazards and risks is one approach to ensuring that children's health is protected. The use of uncertainty factors and children's risk assessment was addressed specifically by Dourson and colleagues when they concluded that a high percentage of the population, including children, is protected when a single uncertainty factor of $10 \mathrm{X}$ is used to account for human variability [36]. Understanding toxicokinetics (TK) and toxicodynamics (TD) in both the test species and in humans provides greater certainty in the risk characterization, and allows for use of chemical specific adjustment factors in lieu of the default values. Conversely, if little is known regarding TK and TD, uncertainty is addressed by use of the default factor of 10X. Unlike pharmaceuticals, TK and mode of action information has typically been developed for commodity chemicals in a higher tier, rather than in the first or screening level tier. However, recent recommendations for implementing a tiered testing approach for pesticides [51] illustrates the value of having such information at hand for design and interpretation of toxicity studies, and as this approach moves forward the lessons learned should be considered for application within the tiered testing of commodity chemicals.

More data on the particular sensitivities of children to chemicals and on unique exposures to children is a welcome addition to the current database of information on chemical toxicities and risks. However, the value and relevancy of the type of toxicity data already available, and currently being gathered for HPV chemicals, should not be discounted in the assessment of potential impacts on susceptible subgroups, in particular children. Recent reviews [35, 36] indicate that the concern expressed by some authors [52] for differential sensitivity of children to chemical toxicity may have been overstated. While the newborn human (less than 6 months of age) may be more sensitive, Scheuplein et al. [35] report that after 6 months of age, children are not usually more sensitive to chemical toxicity than adults, and that in many cases children are less sensitive than adults.
We do understand that concern has been expressed by some that standardized tiered toxicity testing and risk assessment approaches may not provide adequate protection against subtle toxicological effects that could impact infants and children. The concerns are often accompanied by reference to certain examples such as the neurological effects associated with lead exposure. This issue was addressed in an earlier publication [53], in which a retrospective examination of available animal toxicity studies on lead compounds was conducted to assess the adequacy of current animal toxicity testing under regulatory guidelines to protect infants and children. The assessment demonstrated that current guideline animal testing under the Federal Insecticide, Fungicide and Rodenticide Act (FIFRA) would have resulted in setting of an exposure level in humans that is below the current human health regulatory action level for lead in children, without the use of an additional 10X safety factor. In this context, it is important to recognize that the FIFRA test battery for food use pesticide active ingredients is more extensive than the HPV SIDS test battery, and a risk-based safe exposure level derived from the FIFRA dataset would generally employ the typical extrapolation uncertainty factors of $10 \mathrm{X}$ for animals to humans and 10X to account for the most sensitive human (100X composite uncertainty factor). However, additional uncertainty factors would be considered for derivation of a risk-based safe exposure level from HPVlevel toxicity results. Published guidance on use of uncertainty factors for datasets of varying limitations and for datasets that have been judged to be lacking important specialized toxicity studies directs that composite uncertainty factors as high as 3,000 or 10,000 could be applied [54]. However, the guidance also indicates that if a chemical's dataset would be found to require a composite uncertainty factor of more than 10,000 then the data would be considered too uncertain and additional toxicity testing would be sought by EPA. Hasegawa and colleagues [55], based on their direct experimental comparison of repeat dose toxicity studies in newborn rats compared to young animals, concluded that an uncertainty factor of $10 \mathrm{X}$ to account for human variability and a database completeness factor of between $3 \mathrm{X}$ to $10 \mathrm{X}$ would be appropriate to address children's risks "unless knowledge of particular toxicity in newborns or infants is present, or if not present, is discountable due to other credible information on the chemical." By applying these uncertainty factors, including, when appropriate, a database uncertainty factor, risk assessors are able to develop health-based values that are protective of human health, including children's health, even in cases where the toxicity datasets are somewhat limited. Therefore, risk assessment methods currently used in conjunction with tiered testing strategies consider both the quality and the type of available data when extrapolating from data in animals to risks in humans, with consideration of special risks that may be posed to children.

\section{CONCLUSIONS}

From a children's health perspective, data provided as part of the HPV Challenge Program constitutes a wealth of relevant information. The types of studies, as well as the endpoints examined, provide a valuable and appropriate starting point for evaluating potential health hazards to children, as well as adults. The endpoints and observational data 
from the HPV Challenge toxicity testing battery that are directly relevant to evaluating risks to children's health, include: 1) identification and definition of possible hazards upon all major organ systems from both acute and repeated exposures; 2) detection of potential hazards arising from in utero exposures; 3 ) evaluation of potential of a substance to affect reproduction; 4) evaluation of the potential of a substance to damage DNA; and 5) establishment of NOAELs. The acute toxicity studies are most critical to assure correct packaging, labeling and handling to prevent poisoning incidents in children. Developmental and reproductive toxicity studies are most relevant to prevent exposures that could affect normal prenatal and postnatal growth, development and maturation of children. Within a tiered testing and evaluation framework that uses toxicity triggers [5] or in a risk-based paradigm that integrates child-specific exposure data/information (for example, VCCEP) with toxicity data, the hazard characterizations stemming from the HPV Challenge toxicity datasets should inform meaningful screeninglevel risk evaluations for children. The information gained from the HPV Challenge toxicity tests can be used to provide the scientific justification for priority setting and decisions as to whether additional laboratory toxicity tests are warranted, and also which specific tests and endpoints may be pursued to reduce uncertainties or to better define the potential hazard of specific substances.

\section{CONFLICT OF INTEREST}

Statements of fact and opinion expressed in this article are those of the authors. There are no contractual relations or proprietary considerations that restrict dissemination of the research findings of the authors. L. M. Plunkett is an independent consultant. R.A. Becker is employed by the American Chemistry Council, a trade association that represents chemical manufacturers.

\section{ACKNOWLEDGEMENTS}

The authors thank Jim Keith and Steve Russell for their assistance in supplying the HPV test plan information and for their thoughtful review of early drafts of this manuscript.

\section{REFERENCES}

[1] USFDA (US Food and Drug Administration). Regulations requiring manufacturers to assess the safety and effectiveness of new drugs and biological products in pediatric patients. FR63 6663166672, December 2, 1998; Final Rule.

[2] USEPA (US Environmental Protection Agency). Implementing the Food Quality Protection Act, USEPA 735-R-99001; 1999.

[3] WHO (World Health Organization). Principles for Evaluating Health Risks in Children Associated with Exposure to Chemicals. Environmental Health Criteria 237; 2006.

[4] USEPA (US Environmental Protection Agency). Voluntary Children's Chemical Evaluation Program. 65 FR 1699, December 26, 2000.

[5] Becker RA, Plunkett LM, Borzelleca JF, Kaplan AM. Tiered toxicity testing: evaluation of toxicity-based decision triggers for human health hazard characterization. Food Chem Toxicol 2007; 45: 2454-2469.

[6] Landrigan PJ, Carlson JE, Bearer CF, et al. Children's health and the environment: A new agenda for prevention research. Environ Health Perspect 1998; 106(Suppl 3): 787-794.

[7] Goldman LR, Koduru S. Chemicals in the environment and developmental toxicity to children: A public health and policy perspective. Environ Health Perspect 2000; 108: 443-448.
[8] Toxic Ignorance. Environmental Defense Fund 1997. http://www. environmentaldefense.org/documents/243_toxicignorance.pdf

[9] ACC (American Chemistry Council). Public Availability of SIDSRelated Testing Data for US High Production Volume Chemicals; June 12, 1998

[10] USEPA (US Environmental Protection Agency). USEPA's Chemical Hazard Data Availability Study. What do we Really Know About the Safety of High Production Volume Chemicals? The 1998 Baseline of Hazard Information that is Readily Available to the Public. Available from: http://www.epa.gov/hpv/pubs/general/ hazchem.pdf

[11] Faustman EM, Omenn GS. In: Klaassen, CD, Ed, Casarett \& Doull's Toxicology: The Basic Science of Poisons, $6^{\text {th }}$ edition. McGraw-Hill, New York 2001; 83-101.

[12] Renwick AG, Lazarus NR. Human variability and noncancer risk assessment-an analysis of the default uncertainty factor. Regul Toxicol Pharmacol 1998; 27: 3-20.

[13] Pastino GM, Yap WY, Carroquino M. Human variability and susceptibility to trichloroethylene. Environ Health Perspect 2000; 108: 201-214.

[14] IPCS (International Programme on Chemical Safety). ChemicalSpecific Adjustment Factors For Interspecies Differences and $\mathrm{Hu}-$ man Variability: Guidance Document for Use of Data In Dose/Concentration-Response Assessment; 2005. Available from: http://whqlibdoc.who.int/publications/2005/9241546786_eng.pdf

[15] Dourson ML. In: Mertz W, Abernathy CO, Olin SS, Ed. Risk Assessment of Essential Elements. ILSI Press, Washington DC 1994; 51-61.

[16] USEPA (US Environmental Protection Agency). Health Effects Test Guidelines, OPPTS 870.3100 90-Day oral toxicity in rodents, USEPA 712-C-98-199, August 1998.

[17] USEPA (US Environmental Protection Agency). Draft Guidance on Developing Robust Summaries, 1999. Available from: http:// www.epa.gov/hpv/pubs/general/robsumgd.htm

[18] Derelanko MJ, Hollinger MA. CRC Handbook of Toxicology. CRC Press, Boca Raton 1995.

[19] Hayes AW. Principles and Methods of Toxicology. Third edition. Raven Press, New York 1994.

[20] USEPA (US Environmental Protection Agency). OPPTS Harmonized Test Guidelines Series 870 Health Effects Test Guidelines -Final Guidelines. 2007. Available from: http://www.epa.gov/oppts sfrs/publications/OPPTS_Harmonized/870_Health_Effects_Test_ Guidelines/Series/

[21] USFDA (US Food and Drug Administration). Toxicological Principles for the Safety Assessment of Food Ingredients, Redbook. 2000. Available from: http://www.cfsan.fda.gov/ redbook/redtoca.html)

[22] OECD (Organization of Economic Cooperation and Development). Guidelines for the Testing of Chemicals. 2007. Available from: http://www.oecd.org/document/40/0,3343,en_2649_34377_370513 68_1_1_1_1,00.html

[23] USEPA (US Environmental Protection Agency). Toxic Substances Control Act Good Laboratory Practice Standards. 40 CFR Part 792, Thursday, August 17, 1989.

[24] OECD (Organization of Economic Cooperation and Development). GLP Guidelines. 1998. Available from: http://www.olis.oecd.org/ olis/1998doc.nsf/LinkTo/NT00000C5A/\$FILE/01E88455.PDF.

[25] Hodge HC, Sterner JH. Tabulation of toxicity classes. Am Ind Hyg Assoc Q 1949; 10:93.

[26] USEPA (US Environmental Protection Agency). 40 CFR Ch. I (71-99 Edition). 2002. 156.10.

[27] USEPA (US Environmental Protection Agency. (USEPA 2000b). Benchmark Dose Technical Guidance Document. EPA/630/R00/001. Risk Assessment Forum. Washington, D.C. http://cfpub. epa.gov/ncea/cfm/recordisplay.cfm?deid $=42601$

[28] Hiatt GFS, Cogliano VJ, Becker RA, Siegel DM, Den A.Vinyl chloride action levels: Indoor air exposures at a superfund site. In: Andrews JS, Frumkin H, Johnson BL, Mehlman MA, Xintaras C, Bucsela JA Ed, Hazardous waste and public health: International Congress on the Health Effects of Hazardous Waste. Princeton Scientific Publishing, Princeton, New Jersey 1994; 525-529.

[29] Barton HA, Cogliano VJ, Flowers L, Valcovic R, Setzer RW, Woodruff TJ. Assessing Susceptibility from Early-Life Exposure to Carcinogens. Environ Health Perspect 2005; 113:1125-1133. 
[30] Becker RA. Challenges of Implementation of EPA's New Supplemental Guidance for Assessing Cancer Risks from Early Life Exposures. Society for Risk Analysis Annual Meeting, 2005.

[31] USEPA. Supplemental Guidance for Assessing Susceptibility from Early-Life Exposure to Carcinogens, 2005.EPA/630/R-03/003F, http://www.epa.gov/iris/children032505.pdf

[32] Thayer KA, Foster PM. National Toxicology Program Workshop on Hormonally Induced Reproductive Tumors-Relevance of Rodent Bioassays. Environ Health Perspect 2007; 115: 1351-1356.

[33] Wilson JG. Teratology Principles and Techniques. Chicago University Press, Chicago 1965.

[34] Manson JM, Kang YI. In: Hayes AW, Ed. Principles and Methods of Toxicology 2nd ed. Raven Press, New York 1994; 989-1038.

[35] Claudio L, Bearer CF, Wallinga D. Assessment of the US Environmental Protection Agency Methods for Identification of Hazards to Developing Organisms, Part I: The Reproduction and Fertility Testing Guidelines. Am J Ind Med 1999; 35: 543-553.

[36] Claudio L, Bearer CF, Wallinga D. Assessment of the U.S. Environmental Protection Agency Methods for Identification of Hazards to Developing Organisms, Part II: The Developmental Toxicity Testing Guideline. Am J Ind Med 1999; 35: 554-563.

[37] Frankos VH. FDA perspectives on the use of teratology data for human risk assessment. Fundam Appl Toxicol 1985; 5: 615-625.

[38] Jelovsek FR, Mattison DR, Young JF. Eliciting principles of hazard identification from experts. Teratology 1990; 42: 521-533.

[39] Guzelian PS, Henry CJ, Olin SS. Similarities and Differences Between Children and Adults: Implications for Risk Assessment. ILSI Press, Washington DC 1992.

[40] Scheuplein R, Charnley G, Dourson M. Differential Sensitivity of Children and Adults to Chemical Toxicity. I. Biological Basis. Regul Toxicol Pharm 2002; 35: 429-447.

[41] Dourson M, Charnley G, Scheuplein R. Differential Sensitivity of Children and Adults to Chemical Toxicity. II. Risk and Regulation. Regul Toxicol Pharm 2002; 35: 448-467.

[42] USEPA (US Environmental Protection Agency). Health Effects Test Guidelines, OPPTS 870.3050 Repeat Dose 28-Day Oral Toxicity in Rodents, USEPA 712-C-00-366, July 2000. Available from: http://www.epa.gov/opptsfrs/publications/OPPTS_Harmon ized/870_Health_Effects_Test_Guidelines/Series/870-3050.pdf

[43] ACC (American Chemistry Council). Questions and Answers on the Extended HPV Program; 2007. Available from: http://www. americanchemistry.com/s_acc/bin.asp?CID=439\&DID=1509\& DOC $=$ FILE.PDF.
[44] Morford LL, Henck JW, Breslin WJ, DeSesso JM. Hazard identification and predictability of children's health risk from animal data. Environ Health Perspect 2004; 112: 266-271.

[45] Brozena SH. Issues Pertaining to the EPA's EDMVS -Mammalian Multigeneration Reproduction Study Design. USEPA Docket Control Number OPPTS-42212E; 2001.

[46] Olin SS, Sonawane BR. Workshop to develop a framework for assessing risks to children from exposure to environmental agents. Environ Health Perspect 2003; 111: 1524-1526.

[47] Daston G, Faustman E, Ginsberg G, Fenner-Crisp P, Olin S, Sonawane B, Breslin, W. A framework for assessing risks to children from exposure to environmental agents. Environ Health Perspect 2004; 112: 238-256.

[48] Ginsberg G, Slikker W, Bruckner J, Sonawane B. Incorporating children's toxicokinetics into a risk framework. Environ Health Perspect 2004; 112: 272-283.

[49] Landrigan PJ, Kimmel CA, Correa A, Eskenazi B. Children's health and the environment: Public health issues and challenges for risk assessment. Environ Health Perspect 2004; 112: 257-265.

[50] USEPA (US Environmental Protection Agency). A Framework for Assessing Health Risks of Environmental Exposures to Children. USEPA/600/R-05/093F, September 2006.

[51] Barton HA, Pastoor TP, Baetcke K, et al. The acquisition and application of absorption, distribution, metabolism, and excretion (ADME) data in agricultural chemical safety assessments. Crit Rev Toxicol 2006; 36: 9-35.

[52] Landrigan PJ, Mattison DR, Boardman B, et al. Comments on "Children's health susceptibility, and regulatory approaches to reducing risk from chemical carcinogens". Environ Health Perspect 2001; 109: A412-413.

[53] Plunkett L. Do Current FIFRA Testing Guidelines Protect Infants and Children? Lead as a Case Study. Regul Toxicol Pharmacol 1999; 29: 80-87.

[54] Dourson ML. Methods for establishing oral reference doses (RfDs). In: Risk Assessment of Essential Elements, W. Mertz, C.O. Abernathy and S.S. Olin, Ed, ILSI Press, Washington, DC. 1994; 51-61.

[55] Hasegawa R, Hirata-Koizumi M, Dourson M, et al. Pediatric susceptibility to 18 industrial chemicsl: A comparative analysis of newborn with young animals. Regul Toxicol Pharmacol 2007; 47: 296-307.

(C) Plunkett and Becker; Licensee Bentham Open.

This is an open access article distributed under the terms of the Creative Commons Attribution License (http://creativecommons.org/licenses/by/2.5/), which permits unrestrictive use, distribution, and reproduction in any medium, provided the original work is properly cited. 